\title{
EL CANTO DE FEMIO Y LA PROGRESSION DISCURSIVA DE TELEMACO: UN INTERACTO EN COLABORACION
}

Elena Huber

Universidade de Buenos Aires

El canto 1 de Odissea presenta una estructura escindible en cuatro unidades temáticas menores o microestructuras; la primer microestructura está representada por el proemio cuya función metatextual es explícita, la segunda se halla configurada por una breve secuencia narrativa que presenta la localización de Odiseo en la isla de Ogigia, y de Posidón en el país de los etiopes, la tercera contiene las instancias discursivas de la asamblea de los dioses de la cual parten dos decisiones fundamentales: el viaje de Hermes a la gruta de Calipso, y el de Atenea a Ítaca; y, por último, la cuarta microestructura conformada por una secuencia de sucesos que giran en torno a la decisión y preparación del viaje iniciático del hijo de Odiseo.

Este trabajo se centra en la última microestructura de acuerdo con la división del canto 1 de Odissea antes senalada, y tiene como objeto demostrar la existencia de un interacto en colaboración entre el canto de Femio y la progresión discursiva de Telémaco, mediante el análisis de la estructura narrativa comprendida entre los vv. 153-157, en vistas de que previo a la puesta en marcha del viaje en su resolución fáctica son destacables; en el plano del discurso poético, estrategias narrativas que pautan el despliegue de núcleos sémicos ordenadores y funcionalmente constitutivos.

El aporte en cooperación de dos actos individuales para el cumplimiento de un acto alcanzado en común remite al concepto té́rico de interacto en colaboración.

Este tipo de interacción se establece entre dos agentes y esto resulta ser condición necesaria de la existencia de un interacto.

Un agente de acción principal y uno de acción secundaria, 0 ayudante, pueden participar de dicha operación con planes, propositos y motivos diferentes, interactuando con actividades independientes y paralelas que dan como resultado un hecho dependiente de ambos actos.

El contexto en el cual es possible identificar este procedimiento en el planteo de la acción presenta dos situaciones diferenciadas, por un lado, la incorporación de Atenea en el ô̂kos de Odisseo, y, por otro, la realidad subvertida de ese oîkos presentad.

Cabe destacar que la presencia de la divindad no modifica la actitud de los

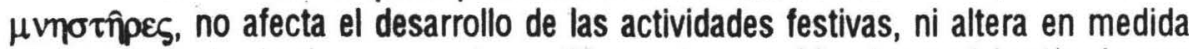
alguna el grado de desmesura de aquéilos; esto se evidencia en el hecho de que Atenea se integra a esa realidad, transfigurada en un anciano apelado Mentes, para cumplir, por una parte, con su cometido esencial que apunta especificamente a modificar la actitud de Telémaco, y, por otra, para que éste pueda por sus propios medios asumir la realidad presente como algo modificable, dado que el proceso de cambio va dirigido hacia pasado vital del hijo de Odiseo limitada por la ca6tica realidad presente. 
Ambos, Atenea-Mentes y Telémaco, se integran al banquete y a partir de esta situación se genera la progresión discursiva de Telémaco en el marco de la actividad de Femio.

Las dos producciones son compatibles en tanto pertenecem al plano de la elaboración verbal del mismo pasado, el referente resulta ser el mismo: la guerra de Troya, los vóotor.

La modalidad respecto de la enunciación es la que diferencia a estos dos discursos paralelos: el de Femio de alcance público ejerciendo, silenciado y en la estructura profunda, tensión sobre el otro, el de Telémaco de carácter privado de acuerdo com las circunstancias de su emisión, pero textualizado en el discurso poético de Odisea.

A partir de la distribución de los lexemas en el sintagma, y de sus relaciones paradigmáticas se propone un análisis sémico de la siguiente estructura que permite identificar la estrategia narrativa de doble proyección simultánea de las actividades discursivas mencionadas.

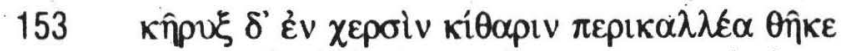

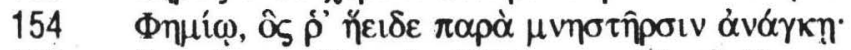

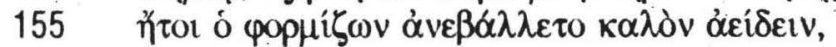

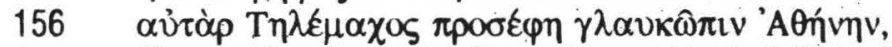

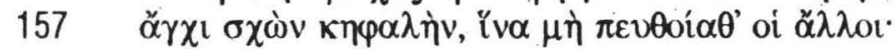

La ubicación central del verbo óvoßád $\lambda \lambda \omega$ en el verso 155 pauta la organización interna de correspondencias sémicas entre los dos sucesos representados sobre esta superficie narrativa; dieho lexema ó $\gamma \varepsilon \beta \alpha \alpha \lambda \lambda \varepsilon \tau$ to articula el

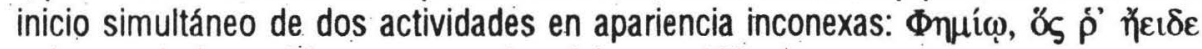

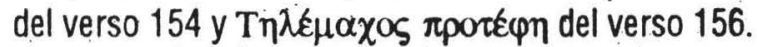

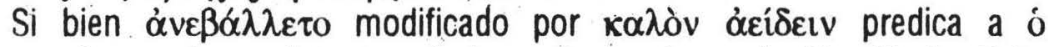

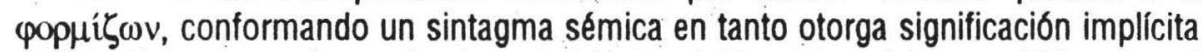

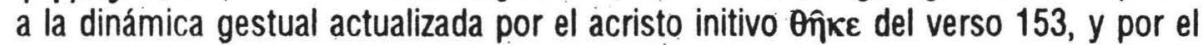

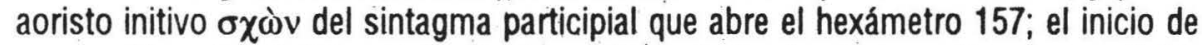

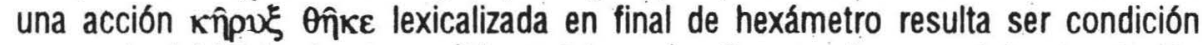

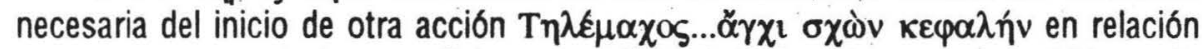
oblicua respecto de la superficie, y ancabalgada a la primera, verso 153, en la estructura profunda. De modo que la estructura en su totalidad resulta un bloque compacto cuyo componente basal es una tríada de acción en imperfectos: $145-\dot{\alpha} v \varepsilon \beta \dot{\alpha} \alpha \lambda \varepsilon \varepsilon \tau 0-\pi \rho 0 \sigma \varepsilon \hat{\varepsilon} \eta$ ajustada a un presente histórico respetando el tiempo de la narración.

Por lo tanto $\alpha v \varepsilon \beta \alpha \lambda \lambda \varepsilon \tau$ to es lexema central de la superficie narrativa al mismo tiempo que ocupa el lugar central en la tríada de sección interior w. 154155-156 del bloque lexémico.

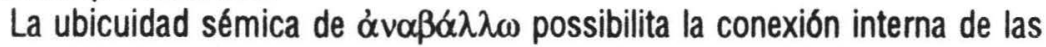

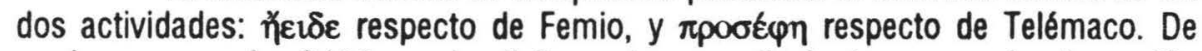
modo que en $\alpha \dot{\alpha} \varepsilon \beta \not \alpha \lambda \lambda \varepsilon \tau \tau$ implicitamente se articula la secuencia de acción precedente vv. 153-154 con la secuencia de acción contigua vv. 156-157, una como condición necesaria de la existencia de la otra; en consecuencia, todo el sistema léxico es absorbido, en el plano semántico, por una fuerza centrípeta ejercida por el 
verbo $\alpha \operatorname{vi} \alpha \alpha_{\alpha} \lambda \lambda \omega$ al incidir éste sobre la activación de las dos elaboraciones discursivas que atrae sobre si en forma yuxtapueste.

En la secuencia comprendida entre los vv. 153-154 están implicados dos agentes; un agente del cual se infiere la producción de una orden representado por

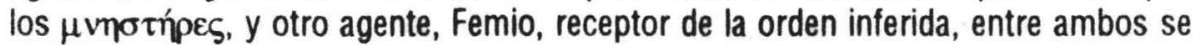
halla un operador intermedio representado por el кńpós.

Las actividades desempeñadas por el кńpú̌ son siempre de carácter público; su intervención responde al cumplimiento de órdenes impartidas por un ǒv $\alpha \xi$. Por lo tanto, es detectable aqul un importante indicio de subverción de roles y una prueba eficiente de caótica inversión del poder en ĺtaca, dado que el $\kappa \eta ́ p u \xi \xi$ transfiere, en esto estadio, una orden no impartida por un ǎvok, sino por los

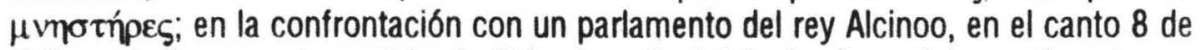
Odisea, halla su contrapartida simétrica la actitud del кńpu’́ que intermedia entre un

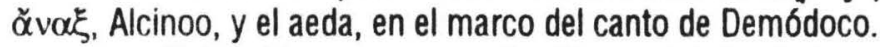

El haraldo asume una función social de destacada importancia en los Poemas Homéricos; es fundamental su rol de portavoz, y dentro de sus actividades específicas cumple la función de habilitante respecto del plano discursivo dado que a él se le comisiona tanto el hecho de depositar la citara en las manos del seda cuanto el cetro en las manos de quien en el ágora tome la palabra. Por estas actividades asociadas a la reproducción verbal, se lo considera descendiente de Hermes de quien goza protección.

Kńpvȩ́ halla se fuente etimológica en karu; del sánscrito: cantor, poeta. En consecuencia tanto el $\kappa n ́ p v \xi \xi$ como el aeda se desplazan sobre el mismo campo semántico, apuntando al acto de habla que en un caso involucra reproducción, y, en otro, producción de materia informativa y de alcance público que proviene de una orden previamente impartida.

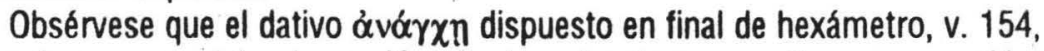
cierra la primer secuencia de acción de la estructura narrativa en cuestión, modificando, por un lado, a Femio en forma sintagmática dentro de la proposición subordinada relativa, y, por otro, en la estructura profunda, en forma paradigmática,

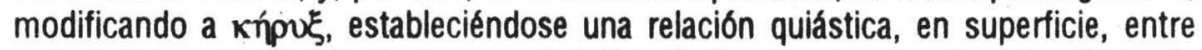
ambos lexemas. Por to tanto, en el dativo óvóryxn se concentra el estado de

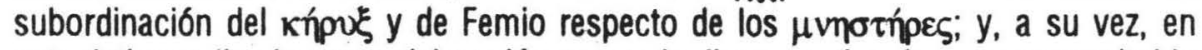
este dativo radica la causa del perdón que se le dispensa el aeda, una vez restituido

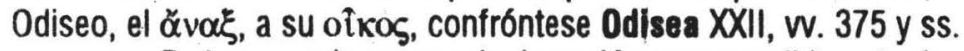

En la segunda secuencia de acción comprendida entre los versos 156-157 se halla implicado un solo agente, Telémaco. El receptor del acto, acto de habla, es

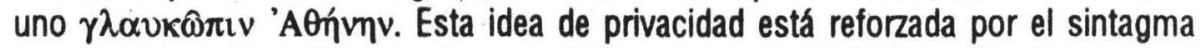

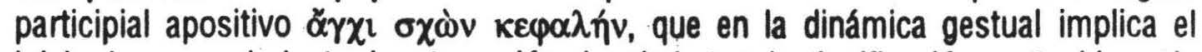
inicio de un movimiento de retroacción vinculado con la significación contenida en la subordinada adverbial final dependiente del núcleo del sintagma participial apositivo.

oi óơ̌ $\lambda \lambda$ a del verso 157, al cerrar el bloque lexémico, por una parte, establece una asociación sémica entre la secuencia de acción cuyo agente es Telémaco, y el dativo óvór $\gamma \mathbf{k} n$ que, como ha sido observado, modifica sintagmáticamente a Femio y paradigmáticamente al $\kappa$ ṕp

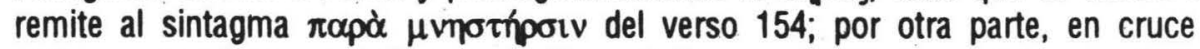
oblicuo ascendente, se halla en relación quástica con T $\eta \lambda \varepsilon \mu \alpha \chi \propto \varsigma$ del verso 156,

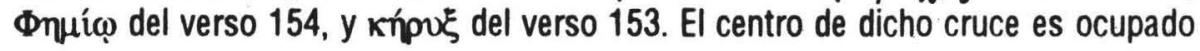


por la tríada de acción en imperfectos

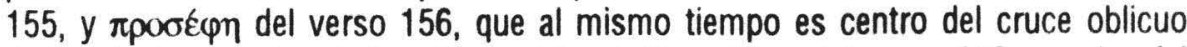

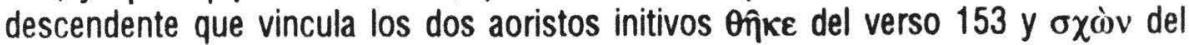
verso 157, actualizándose así la dinámica gestual de cada una de las secuencias de acción proyectadas en esta superficie, que en virtud del lexema óvóx $\gamma$ n apuntan, una, la primera, a la difusión pública, y, la otra, a la reserva y la privacidad.

Por lo tanto, de acuerdo con este análisis, el verbo ávoßá $\lambda \lambda \omega \omega$ del verso 155 , cualquiera sea la perspectiva sémica que se adopte, es punto de confluencia de un interacto en colaboración, Telémaco como agente prinecipal, y, Femio, como agente auxiliar 0 ayudante.

Merece ser destacado el hecho de que tres de los núcleos constitutivos de la superficie narrativa analizada contienen una carga semántica univoca: la palabra. En el caso del $\kappa n ́ p u \xi$, asumida como una función social, como algo inherente, en el caso de Femio, y respecto de Telémaco, como algo necesario. Estas tres instancias en armonía se contraponen al descontrol focalizado en $\pi \alpha$ ò

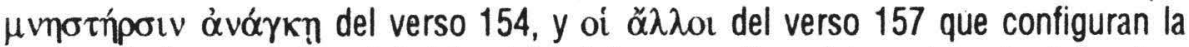
carga sémica negativa inferida del núcleo agentivo del cual parte la orden:

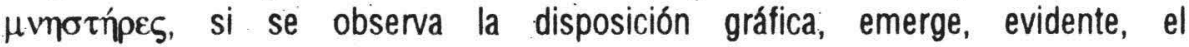
enfrentamiento.

Los aedas ocupan una posición social intermedia dado que eran respetados tanto por parte del pueblo como por parte de la aristocracia; un estudio de Z. Ritook ${ }^{1}$, a través del seguimiento de los epitetos que en Odisea acompañan las menciones de los aedas, demuenstran que el rapsoda gozaba de un estatus privilegiado dentro de la sociedad homérica. F. Bertolini ${ }^{2}$, analizando un pasaje de Odissea XVII, señala la importancia de esta profesión sobre las mencionadas en los vv. 384 y 385 : vate, médico, carpintero, dado que, en abstracción, éstas últimas son para Homero funciones de la poesía; si bien son distintas y autónomas entre sí, son solidarias con ella, y, al mismo tiempo se hallan emparentadas por cuanto que las cuatro profesiones gozan de la protección de Apolo. F. Pfister ${ }^{3}$, a proposito de Odisea VIII, v. 499, se refiere a la antigua creencia de un estado poseso o de inspiración divina respecto de los poetas, tema que en la antigüedad fue abordado

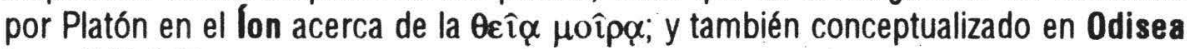
I, vv. 347-349:

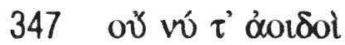

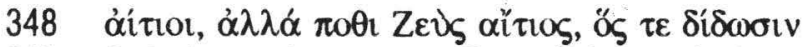

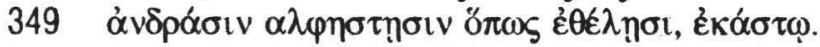

Respecto de la función del canto en los poemas homéricos, Lloyd ${ }^{4}$, a propósito de Odisea I, vv. 353-355 y VIII, vv. 152-157, señala que el canto del aeda no sólo cumple una función didáctica, moralizante o de esparcimiento, sino que 10

1. RITOOK, Z. The epithets for minstrels in the Odissey. A Ant Hung XVI, 1968, 291-346.

2. BERTOLINI, F. Odisseo aedo, Omero carpentiere, Od., XVII, 384-385. Lexis, 1988, 145-164.

3. PFISTER, F. Zwei Sprachlich-Religions. Geschichtliche Bermerkungen zu Homer. WJA III, 1948, 406408.

4. LLOYD, M. Homer on poetry. Two passages in the Odissey. Eranos LXXXV, 1987, 85-90 
que prevalece y resulta más importante es la autenticidad que brinda a los hechos heróicos; G. A. Samoná ${ }^{5}$ destaca que la importancia del canto del aeda en los poemas homéricos radica en la posibilidad que brinda sólo el canto para salvaguardar al héroe del olvido y conferirle inmortalidad; por otra parte, Scully ${ }^{6}$, a partir de un análisis de Odisea III, wV. 263-272, aborda el tema del aeda desde el punto de vista social en relación a el aeda custodio de Clitemnestra, destacando el oder de la poesía oral sobre el auditorio, y su función ordenadora operando sobre los cánones de la conducta humana en sociedad.

Lo relevado sucintamente acerca de estado de la cuestión indica que la crítica ha investigado el tema de los aedas desde el punto de vista social, religioso, o metapoético como es el caso del estudio de E. Diehl ${ }^{7}$, "Fuerunt ante Homerum poetae", 0 el de R. Sealey ${ }^{8}$, "From Phoemios to Ion."

Respecto de la funcionalidad del aeda como personaje de un oíkos en orden, y su canto, objeto de mayor interés por parte de la crítica; no así la presencia de Femio, el aeda de un oîxos en desorden.

Eustacio en Ad Odyss. 1.155, refiriéndose a Femio dice:

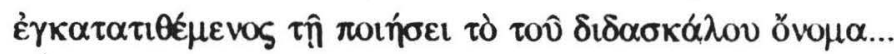

esta indicación resulta de corte metapoético, como también lo evidencia

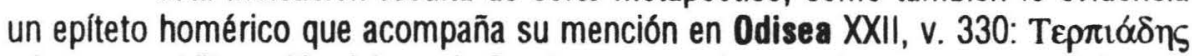
y luego una afirmación del propio Femio respecto de su actividad en Odisea XXII, v.

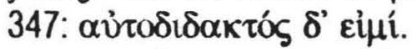

La mencion del aeda Femio es acompañada por otros dos epítetos

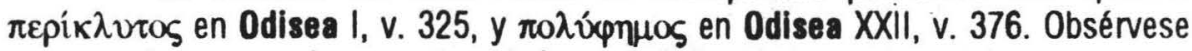
que en este caso, ambos apuntan al alcance público de lo que le es inherente, como culminación semántica de su nomore o profusión del mísmo cuya raíz etimológica proviene del verbo $\varphi \eta \mu$ í.

El análisis que se ha propuesto de la estructura narrativa antes desarrollado, permite detectar la función agentiva de Femio como personaje secundario cooperador respecto de la acción principal desplegada entre los vv. 153371 de Odisea I.

El canto de Femio propicia una situación de orden momentáneo en el ô̂xos de Odiseo. La reestructuración, aparente, del código de comportamiento social que Femio como aeda tiene el poder de lograr al convocar la máxima atención de su auditorio, posibilita el inicio de la progresión discursiva de Telémaco, colaborando en tanto que evita que se produzea una fisura entre esta situación y su contexto - confróntese Odisea I, vv. 161-165: Telémaco abre su discurso con una

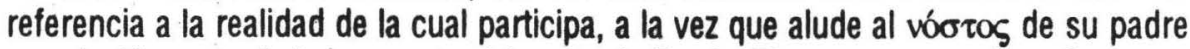
en relación metonímica respecto del canto de Femio. El nexo entre ambos discursos es el referente: los vóotot.

5. SAMONA, G. A. L'aedo metieta nascosto nell Illada e la moira di Achille. SMSR VII, 1983, 161-182.

6. SCULLY. The bard as the custodian of homeric society, Od. III, 263-272. QUCG, 1981, 37, 67-83.

7. DIEHL, E. Fuerunt ante Homerum poetae. Rh. M., 1940, 81-114.

8 SEALEY, R. From Phoemios to Ion. REG, tome LXX, 1957 
Mientras que Femio impene un orden exterior, Telémaco reclama un orden interno que le es suministrado por medio de un trayecto dialógico y reflexivo que lo conduce a la reconstrucción de la figura paterna y a la recuperación interior del ǒvoß̌ de ĺtaca, Odiseo.

La interrupción de Penélope, Odisea I, vv. 336-344, provoca la culminación simultánea de las dos actividades discursivas proyectadas, también, en forma simultánea, en Odisea I, vv. 153-157.

Es en este punto donde el interacto en colaboración entre el canto de Femio y la progresión discursiva de Telémaco halla su justificación teórica y su fundamento textual en el plano del discurso poético de Odisea.

Femio, en calidad de agente auxiliar 0 ayudante, es componente del interacto; esto de por sl implica la existencia de una función agentiva en el aeda como personaje; resulta componente en tanto que colabora con Telémaco en el interacto que halla su materialización en la secuencia de sucesos de la cual participan el aeda, Penélope, y el hijo de Odiseo, secuencia contendida entre los versos 336-380.

La actividad de Femio resulta ser condición necessaria para que se genere la progressión discursiva de Telémaco en tanto que dicha progresión resulta ser condición necessaria para que, por una parte, pueda ser enaltecida la actividad de Femio, Odisea I, vv. 570-371, y por otra, para que el aeda continue cantando ya no por decisión de los $\mu$ vmoтńpes sino por lá justificación de Telémaco, Odisea I, vv. 350-355:

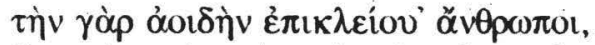

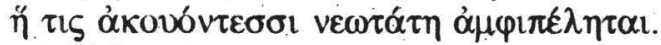

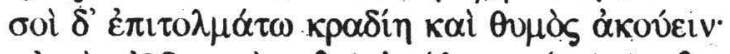

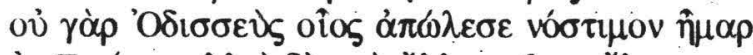

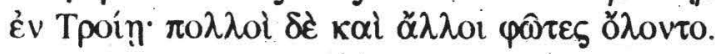

La boole proyección simultánea es funcional puesto que permite establecer una lógica interna respecto de la acción a través de las dos producciones verbales como dos instancias de armonía, en un caso frente a los $\mu$ viotńpes en función de Telémaco, y en otro frente a la $\beta \alpha \sigma i \lambda \varepsilon$ é $\alpha$ en función del aeda.

La realidad sobre la que se han desplazado las dos actividades continúa siendo la mesma, pero a partir de la culminación de éstas se desprende el primer intendo humano en cooperación por modificarla.

\section{Bibliografia}

ChANTRAine, P. Dictionnaire étymologique.

FINLEY, M. I. El mundo de Odisseo. México, FCE, 1978.

GRIFFIN, J. Homero. Madrid, Alianza Ed., 1984.

PAUL BONET, J. Los heraldos, Taltibio y Eurípides. Helmántica VII, 1956.

PAULYS REAL. Encyclopadie der Classischen.

RODRÍGUEZ ADRADOS y otros. Introducción a Homero. Barcelona, Ed. Labor, 1984.

ROSCHER, W. H. Lexicon der Mythologie III, 2. Leipzig, 1902-1904.

STEPHANUS. Thesaurus graecae Linguae.

VAN DIKJ, T. A. Texto y contexto. Madrid, Cátedra, 1980. 